\title{
COUNTER-ROTATING MOLECULAR GAS IN NGC 4546
}

\author{
LESLIE J. SAGE \\ Max-Planck-Institut für Radioastronomie, Auf dem Hügel 69, 53121 Bonn, Germany, Department of Physics, University of \\ Nevada-Las Vegas, Las Vegas, Nevada 89154, and Department of Astronomy, University of Maryland, College Park, Maryland \\ 20742 \\ Electronic mail: 1sage@astro.umd.edu
}

\section{GIUSEPPE GALLETTA}

Dipartimento di Astronomia, Università di Padova, Vicolo Osservatorio 5, 35122 Padova, Italy

Received 1994 May 4; revised 1994 June 14

\begin{abstract}
The detection of $9.6 \pm 2.9 \times 10^{7} \mathscr{L l}_{\odot}$ of molecular gas in the barred S0 galaxy NGC 4546 is reported. This galaxy has an $8 \mathrm{kpc}$ wide disk of ionized gas rotating opposite to the stellar disk (but with approximately the same velocity magnitude). The detected molecular gas has the same motion as the ionized gas (and the atomic gas), counter-rotating with respect to the stars. The molecular gas is concentrated more on the western side of the galaxy, while the atomic gas is apparently more prevalent on the eastern side. The peculiar gas kinematics of NGC 4546 likely come from a past acquisition of gas, probably through a collision with a dwarf galaxy whose relative velocity was retrograde with respect to the galaxy spin. However, the lack of evidence for starburst activity, and the length of time required to form a stable disk structure from an accreted companion, suggest that there is little interaction between the pre-existing and accreted gas at this time.
\end{abstract}

\section{INTRODUCTION}

Although it looks like a normal SB0 galaxy seen edge-on, NGC 4546 is actually a system in which the peculiar phenomenon of gas counter-rotation is seen (Galletta 1987). It contains a stellar disk with a rotational velocity of $\sim 250$ $\mathrm{km} \mathrm{s}^{-1}$ and a disk of ionized gas (radius $\sim 8 \mathrm{kpc}$ ) spinning at a similar speed, but in the opposite direction to the stars. The phenomenon is so evident that it is possible to see on the same optical spectrum the emission and absorption lines inclined in opposite ways. The mean nuclear velocity of this disk of gas is $1014 \pm 35 \mathrm{~km} \mathrm{~s}^{-1}$, not very different from that of the stars, $1069 \pm 24 \mathrm{~km} \mathrm{~s}^{-1}$, or the $\mathrm{H} \mathrm{I}, 1050 \mathrm{~km} \mathrm{~s}^{-1}$. This indicates that we are observing a structure inside the galaxy, and not a separate gaseous component along the same line of sight as the optical image.

The counter-rotation of the gaseous component can be explained if this galaxy, as has been suggested for NGC 5128 and other systems, has accreted its gas from outside, possibly through the capture and destruction of a satellite. The nucleus of such a satellite may be the structure seen in $\mathrm{H} \alpha$ light as a bright cloud, $175 \mathrm{pc}$ wide, slightly offset from the stellar nucleus of NGC 4546 (Bettoni et al. 1991).

Following the detection of counter-rotating gas in NGC 4546 , other cases of gas counter-rotation have been found among S0s (see, e.g., Rubin 1988; Dettmar et al. 1990; Bertola et al. 1992). Many cases of gas counter-rotation are also known among elliptical galaxies (Bettoni 1984; Caldwell et al. 1986; Bertola \& Bettoni 1988; Schweizer et al. 1989; Bertola et al. 1990), while in the spiral galaxy NGC 4826 atomic gas in counter-rotation has been observed (Braun et al. 1992; van Driel \& Buta 1993). These galaxies generally lack obviously interacting companions, which suggests that the source of dust and ionized gas has been completely disrupted.

NGC 4546 presents still more peculiarities. On one hand, the intensity of forbidden lines smoothly decreases from the center outward, indicating a uniform structure for the ionized gas. On the other hand, the $\mathrm{H} \alpha$ emission line is often absent on the eastern side of the galaxy (the major axis runs almost east-west, P.A. $\left.=78^{\circ}\right)$. The cool gas also seems to be asymmetric. H I $(21 \mathrm{~cm})$ observations at the Westerbork Synthesis Radio Telescope (WSRT) indicate that on the east side $\mathrm{HI}$ is more abundant than on the west side (Bettoni et al. 1991).

This second peculiarity opens many interesting questions: if the gas is not coeval with stars, but belongs to a second event in the history of the galaxy, does its distribution reflect the collision symmetry, rather than the galaxy symmetry? Can the accreted gas, now moving at $\sim 350 \mathrm{~km} \mathrm{~s}^{-1}$ with respect to the stars, continue to travel inside the galaxy without further interaction, with either the stars or pre-existing gas? If some gas were already present in NGC 4546 at the time of the collision, has this material been heated and subsequently cooled through dust and molecular emission, or is it still interacting with the accreted matter?

To examine further this peculiar gas distribution, we observed the lowest rotational transitions of $\mathrm{CO}(J=1 \rightarrow 0$, $J=2 \rightarrow 1)$ to trace the $\mathrm{H}_{2}$ within the galaxy. The results of this study are presented in the following sections, along with some suggestions as to interpretation.

\section{OBSERVATIONS AND DATA ANALYSIS}

The data were obtained during four separate observing runs, 1990 June 23-25, 1993 Feb 12, and 1993 July 8-10 at 

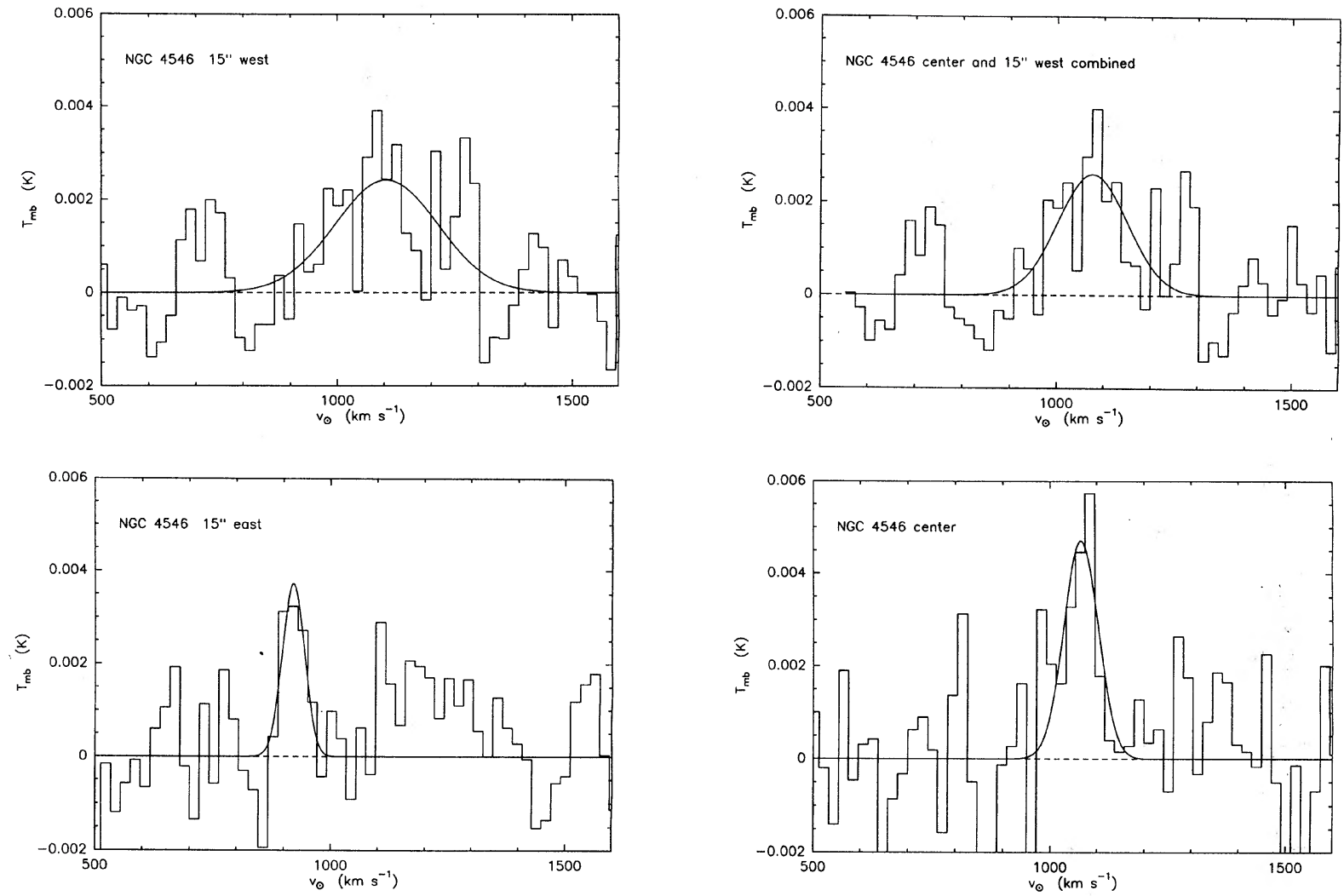

FIG. 1. The CO $1 \rightarrow 0$ spectra obtained at the NRAO $12 \mathrm{~m}$ telescope. Temperatures are on the Rayleigh-Jeans main beam brightness scale $\left(T_{\mathrm{mb}}\right)$. The positions of each beam on the galaxy are indicated on the spectra, where "west" corresponds to an offset of $(\delta$ R.A., $\delta$ Decl. $)=\left(-15^{\prime \prime},-3\right.$ "), relative to the center position [R.A.(1950) $=12: 32: 54.9$, Decl.(1950) $=-03: 31: 04]$, while "east" denotes an offset of $\left(-15^{\prime \prime},-3^{\prime \prime}\right)$. The offset positions lie on the major axis of NGC 4546 . One panel shows the sum of the central and western pointings.

the NRAO ${ }^{1}$ 12-m telescope at Kitt Peak, and 1991 November 1-3 at the IRAM $30 \mathrm{~m}$ telescope at Pico Veleta, Spain.

\subsection{NRAO 12 m Telescope}

The $12 \mathrm{~m}$ telescope has a beam size (HPBW) of $55^{\prime \prime}$ at $115 \mathrm{GHz}$. The dual-polarization $3 \mathrm{~mm}$ SIS receivers were utilized in conjunction with the 256 channel $\times 2 \mathrm{MHz} /$ channel filterbanks. System temperatures were typically between 850 and $1100 \mathrm{~K}$ on the Rayleigh-Jeans main beam brightness temperature scale $\left(T_{\mathrm{mb}}\right)$ for the 1990 run, and $\sim 500 \mathrm{~K}$ during the 1993 runs. Telescope pointing and focus were checked frequently using the nearby source 3C 273. Because $3 C 273$ is so close to NGC 4546 in the sky, we estimate the pointing uncertainty to be $\sim 4^{\prime \prime}$.

Observations of the $\mathrm{CO} J=1 \rightarrow 0$ line were made at three locations in NGC 4546, the center [R.A. $(1950)=12: 32: 54.9$, Decl.(1950) $=-03: 31: 04]$ and two positions $15^{\prime \prime}$ away from the center along the major axis (on opposite sides). The data were obtained using the double-beam-switching mode, with a $4^{\prime}$ beam throw in azimuth (BSP). All scans at each position were coadded, Hanning smoothed and replotted with a channel spacing of $\sim 21 \mathrm{~km} \mathrm{~s}^{-1} /$ channel. A linear baseline was

${ }^{1}$ The National Radio Astronomy Observatory is operated by Associated Universities, Inc., under cooperative agreement with the National Science Foundation. then subtracted, producing the spectra displayed in Fig. 1. In addition, all scans at the center and western positions were coadded, smoothed and baselined, with the result also shown in Fig. 1. The final integrated intensities for each position are listed in Table 1.

The utility of observing positions separated by only $1 / 4$ beam may not seem obvious at first glance. However, as demonstrated by Sage \& Isbell (1991), a difference of only $12^{\prime \prime}$ in the position can make a large difference in the spectrum. As we expect the molecular gas to be restricted to a region fairly near the center of the galaxy, a $15^{\prime \prime}$ offset was deemed to be sufficient to determine the sense of gas rotation.

\subsection{IRAM 30 m Telescope}

The HPBW of the $30 \mathrm{~m}$ telescope is $22^{\prime \prime}$ at $115 \mathrm{GHz}$ and $12.5^{\prime \prime}$ at $230 \mathrm{GHz}$. Observations of the $\mathrm{CO} J=2 \rightarrow 1$ and

TABLE 1. CO observations of NGC 4546.

\begin{tabular}{lllll}
\hline \hline Telescope & $\begin{array}{l}\text { Offset } \\
\left({ }^{\prime \prime},{ }^{\prime}\right)\end{array}$ & Transition & $\begin{array}{l}\text { Intensity } \\
\left(\mathrm{K} \mathrm{km} \mathrm{s}^{-1}\right)\end{array}$ & $\begin{array}{l}\text { Centroid } \\
\left(\mathrm{km} \mathrm{s}^{-1}\right)\end{array}$ \\
\hline NRAO 12m & $(0,0)$ & $1 \rightarrow 0$ & $0.50 \pm 0.19$ & $1085 \pm 10$ \\
& $(15,3)$ & & $0.22 \pm 0.12$ & $908 \pm 10$ \\
& $(-15,-3)$ & & $0.68 \pm 0.12$ & $1105 \pm 21$ \\
& $(0,0)+(-15,-3)$ & & $0.54 \pm 0.10$ & $1076 \pm 17$ \\
IRAM 30m & $(0,0)+(-10,-2)$ & $2 \rightarrow 1$ & $2.22 \pm 0.25$ & $1165 \pm 2$ \\
& & $1 \rightarrow 0$ & $1.20 \pm 0.28$ & $1090 \pm 34$ \\
\hline \hline
\end{tabular}




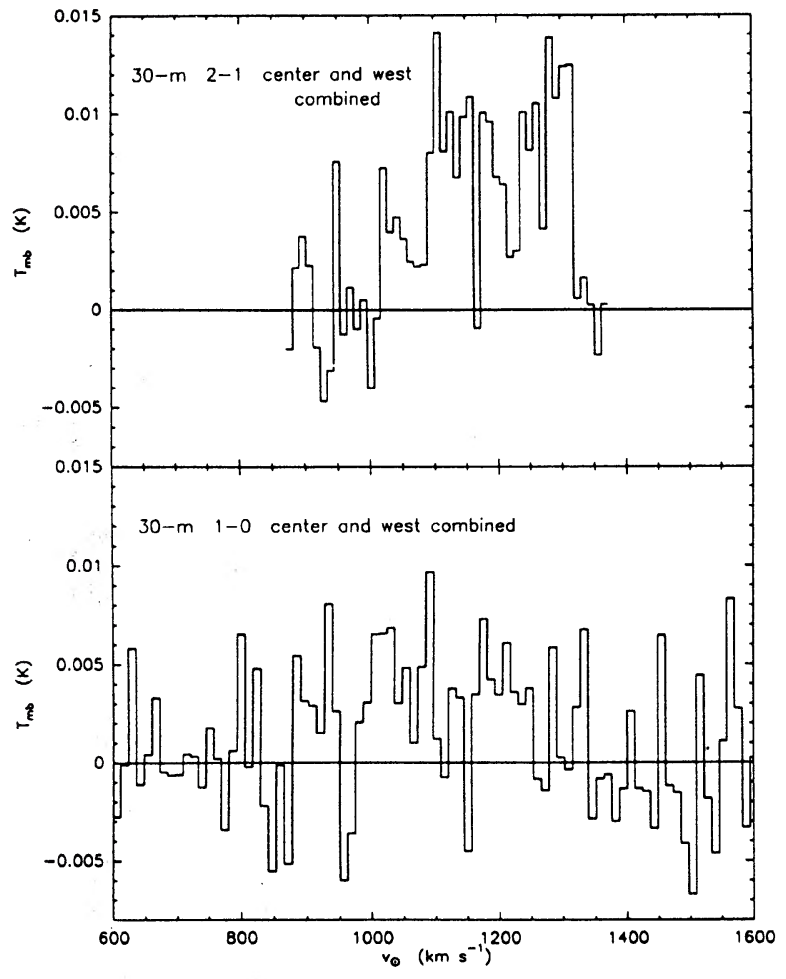

FIg. 2. The IRAM 30-m data at both the $1 \rightarrow 0$ and $2 \rightarrow 1$ transitions. The data obtained at both the central and western $(\delta$ R.A., $\delta$ Decl. $)=\left(-10^{\prime \prime},-2^{\prime \prime}\right)$ pointings have been coadded for both transitions. Temperatures are on the main beam brightness scale.

$1 \rightarrow 0$ lines were made using the $1.3 \mathrm{~mm}$ and $3 \mathrm{~mm}$ SIS receivers with, respectively, the 512 channel $\times 1 \mathrm{MHz} /$ channel filterbank and the acousto-optical spectrometer ( 864 channels $\times 0.585 \mathrm{MHz} / \mathrm{channel}$ ). System temperatures were typically about $1500 \mathrm{~K}$ at $230 \mathrm{GHz}$ and $1100 \mathrm{~K}$ at $115 \mathrm{GHz}$, on the Rayleigh-Jeans main beam brightness temperature scale $\left(T_{\mathrm{mb}}\right)$. Telescope pointing and focus were checked frequently, again using 3 C 273. The data were obtained using double beam switching, with a $4^{\prime}$ beam throw in azimuth (WSW).

Two positions were observed, the center and $10^{\prime \prime}$ west along the major axis, and the data were reduced in a similar way to those from the $12 \mathrm{~m}$ telescope. The one difference is in the final channel spacing of the reduced spectra, which here is $\sim 11 \mathrm{~km} \mathrm{~s}^{-1}$. Because of the poorer signal to noise in the IRAM results, and since the beam is much smaller than that of the $12 \mathrm{~m}$ telescope, the data from both positions were averaged. This is true of both transitions. The final spectra are displayed in Fig. 2, while the integrated intensities are listed in Table 1. Although the determination of a baseline for the $2 \rightarrow 1$ spectrum seems uncertain, the very flat baselines produced by the "wobbler" at that telescope, along with the line emission apparently ending at $\sim 1320 \mathrm{~km} \mathrm{~s}^{-1}$, about the same as for the $1 \rightarrow 0$ line observed at the $12 \mathrm{~m}$ telescope, give us some confidence in the result.

The uncertainties in the line intensities (Table 1 ) were calculated in the standard way (see e.g., Sage \& Wrobel
1989), while the uncertainties in the centroid velocities were determined from Gaussian fits to the data.

\section{RESULTS}

\subsection{Characteristics of the CO Emission}

Weak CO emission was detected at all observed positions. At the center and western positions the $\mathrm{CO}$ emission is located at velocities that are mostly higher than systemic, although the summed spectra may show emission down to $\sim 900 \mathrm{~km} \mathrm{~s}^{-1}$ (Figs. 1 and 2). The eastern position (Fig. 1) shows $\mathrm{CO}$ emission between $\sim 880$ and $\sim 980 \mathrm{~km} \mathrm{~s}^{-1}$, with the possibility of additional emission at higher velocities (1100 to $1320 \mathrm{~km} \mathrm{~s}^{-1}$ ). The most striking aspect of the spectra is that the emission is not symmetric about the systemic velocity obtained from optical absorption lines, with more emission at higher velocities. The $\mathrm{CO}$ emission also seems to go to slightly higher velocities than the $\mathrm{H} \mathrm{I}$ emission [the highest velocity observed by Richter \& Huchtmeier (1987) is $\sim 1250 \mathrm{~km} \mathrm{~s}^{-1}$ ], but may not extend down to the $\sim 850$ $\mathrm{km} \mathrm{s}^{-1}$ seen in the $\mathrm{H}$ I line, although the noise in the both the $\mathrm{CO}$ and $\mathrm{H}$ I data makes the positions of the line edges rather uncertain. $\mathrm{H}$ I was detected on the eastern side of NGC 4546 when observed with an interferometer, but not on the western. This seems consistent with the differences between the $\mathrm{H} \mathrm{I}$ and $\mathrm{CO}$ single-dish spectra.

This asymmetry in the phase ( $\mathrm{H}$ I vs molecular) of the gas is unlikely to arise from errors in pointing, because of the good agreement between the velocities of the ionized gas, and $\mathrm{H} \mathrm{I}$ and $\mathrm{CO}$ emission. Most of the $\mathrm{H} \mathrm{I}$ emission seen in the WSRT map on the eastern side of the galaxy, is at velocities between 850 and $1000 \mathrm{~km} \mathrm{~s}^{-1}$, in agreement with the $\mathrm{CO}$ emission on that side. The ionized gas velocities, as determined from the optical spectra (Bettoni et al. 1991), range between 850 and $1000 \mathrm{~km} \mathrm{~s}^{-1}$ on the $E$ side, in good agreement with the $\mathrm{H} \mathrm{I}$ map, and from 1000 to $1200 \mathrm{~km} \mathrm{~s}^{-1}$ on the W side, similar to the CO spectra towards that side. Additionally, $\mathrm{H} \alpha$ emission, associated with massive young stars, is strongest from the western side, so it is physically reasonable that the molecular gas, out of which the stars form, should be more abundant there.

The $\mathrm{CO}$ is probably fairly centrally concentrated, because the emission detected at the $30 \mathrm{~m}$ telescope is stronger than that detected at the $12 \mathrm{~m}$, as would be expected if the $\mathrm{CO}$ source were smaller than the $55^{\prime \prime}$ beam at the $12 \mathrm{~m}$. On the other hand, the gas has some spatial extent because the line centroid shifts with position. In this situation, any comparision of the $2 \rightarrow 1$ and $1 \rightarrow 0$ line intensities is not physically reasonable, and we cannot say anything about the intrinsic line ratio.

\subsection{The Molecular Mass}

The CO flux was estimated as the sum of the integrated intensities of the eastern and coadded center and western positions observed with the $12 \mathrm{~m}$ telescope, after crudely correcting for beam overlap by multiplying the total by 1.05 , the approximate area (in $\operatorname{arcmin}^{2}$ ) of the combined beams. This produces a total CO luminosity of $L_{\mathrm{CO}}=2.0 \pm 0.6 \times 10^{7}$ $\mathrm{K} \mathrm{km} \mathrm{s}^{-1} \mathrm{pc}^{2}$, assuming a distance of $17 \mathrm{Mpc}$. The molecular 
mass was calculated using a $\mathrm{CO}$ to $\mathrm{H}_{2}$ conversion factor calibrated for a sample of virialized molecular clouds

$$
M\left(\mathrm{H}_{2}\right)=4.8 L_{\mathrm{CO}}\left[\mathscr{M}_{\odot} /\left(\mathrm{K} \mathrm{km} \mathrm{s}^{-1} \mathrm{pc}^{2}\right)\right]
$$

(Solomon et al. 1987), resulting in

$$
M\left(\mathrm{H}_{2}\right)=9.6 \pm 2.9 \times 10^{7}\left(\mathscr{C}_{\odot}\right) .
$$

Assigning a meaningful uncertainty to the $\mathrm{H}_{2}$ mass is difficult, however, because the gas physical conditions may differ substantially from those for which the standard conversion factor is derived. In particular, if the $\mathrm{CO}$ is optically thin, then the assumptions which go into determining the standard conversion factor do not apply.

One indication that the calculated molecular mass is not likely to be in error by too much is that the ratio of far infrared luminosity $\left(L_{\mathrm{FIR}}\right)$ to molecular mass is $L_{\mathrm{FIR}} / \mathscr{C l}\left(\mathrm{H}_{2}\right) \simeq 1.7 L_{\odot} / \mathscr{C b}_{\odot}$, similar to that of many spiral and lenticular galaxies (e.g., Solomon \& Sage 1988; Sage \& Wrobel 1989; Sage 1993). This suggests that the $\mathbf{H}_{2}$ mass determined here is only about as uncertain as in other spiral galaxies. The $L_{\text {FIR }}$ (between $\sim 40$ and $120 \mu \mathrm{m}$ ) was estimated from the 100 and $60 \mu \mathrm{m}$ flux densities $\left(S_{100}=0.79 \pm 0.20 \mathrm{Jy}, S_{60}=0.270 \pm 0.046 \mathrm{Jy} ; \mathrm{Knapp}\right.$ et al. 1989).

\section{DISCUSSION}

Figure 1 clearly indicates stronger $\mathrm{CO}$ emission on the western side of NGC 4546 than on the eastern. As discussed in the previous section, this asymmetry is unlikely to arise from errors in pointing; rather, it indicates that the $\mathrm{H}_{2}$ clouds are present mainly on the western side of NGC 4546, where the gas velocities are higher than systemic, while stars have lower than systemic velocities on that side. The difference between the gas and stellar velocities supports the idea that the detected $\mathrm{CO}$ emission originates in gas that is counterrotating with respect to the stars, and thus was originally accreted from outside the galaxy. This is the first case where molecular gas has been detected in counter-rotation with respect to the stars, although Sage \& Galletta (1993) found molecular gas rotating perpendicular to the stars in three dust-lane ellipticals and Combes et al. (1992) observed CO gas in the polar-ring galaxy NGC 660. As we did for those ellipticals, we suggest that the counter-rotating gas in NGC 4546 was accreted from a dwarf companion, which has now been fully absorbed into the galaxy.

The H I WSRT map (see Fig. 2 of Bettoni et al. 1991) shows the peak emission ( $\sim 4 \sigma$ above the noise) confined to the eastern side of the galaxy, and located $\sim 1.5^{\prime}$ away from the center (almost a full synthesized beamwidth away). At higher velocities, there is only a marginal detection of $\mathrm{H} \mathrm{I}$, but always east of the nucleus. As the WSRT is likely to observe the densest gas, the higher velocity emission observed with a single-dish telescope probably arises from more diffuse gas. We note, however, that the total mass of $\mathrm{H}$ I detected by the WSRT $\left(\sim 1.2 \pm 0.8 \times 10^{8} \mathscr{M}_{\odot}\right)$, is in reasonable agreement with the mass $\left(1.5 \pm 0.6 \times 10^{8} \mathscr{M}_{\odot}\right)$ calculated from Richter and Huchtmeier's observation. It is interesting that the global ratio of molecular to atomic gas is lower in NGC 4546 than in many early-type spirals (e.g., Sage 1993), but a crude estimate of the ratio on the western side alone suggests that the value may be more normal there.

A concentration of atomic gas on one side of the galaxy and molecular gas on the other side suggests that the gaseous disk of NGC 4546 is nonuniform in some way. It is impossible to determine from the current data exactly what parameters (e.g., gas temperature, density) are changing from one side to the other in the galaxy, but Fig. 4 of Bettoni et al. (1991) clearly shows that dust is more prominent on the western side, where the molecular gas seems to be concentrated. We note that the assumed asymmetry in the $\mathrm{H}_{2}$ distribution depends on a constant conversion factor (across the galaxy) between $\mathrm{CO}$ luminosity and $\mathrm{H}_{2}$ mass. This is generally a reasonable assumption for most galaxies (see, e.g., Sage et al. 1990; Sage et al. 1992; Braine et al. 1993), but it may not be for a system which has recently accreted gas from an external source. Different degrees of mixing of the accreted and pre-existing gas could result in very different chemical compositions, possibly affecting the $\mathrm{CO}$ to $\mathrm{H}_{2}$ conversion factor substantially, but the low ratio of $L_{\mathrm{FIR}} / M\left(\mathrm{H}_{2}\right)$ indicates a lack of starburst activity, which in turn suggests that there is little interaction between any pre-existing gas and accreted gas at this time. Moreover, it is difficult to conceive of a regular disk which is fast rotating but composed of different kinds of matter on the two sides. The almost circular rotation seen by Bettoni, Galletta, and Osterloo, the elongation of the retrograde orbits perpendicular to the bar [visible from the velocity field; Galletta (1987)], and the position of the turning point in the gas rotation curve (at the same radius as the stellar rotation curve), all suggest that the gravitational potential of this barred S0 has already shaped the ionized gas into a disk-like configuration. Rotational periods for gas clouds range from 62 million years at $1.5 \mathrm{kpc}$ to 164 million years at $4 \mathrm{kpc}$. This would seem to imply that the material in the inner regions is already well mixed, since the time needed to form a disk from an accreted satellite is larger than these orbital periods (see Rix \& Katz 1991), but that leaves the explanation of the asymmetry very unclear. More detailed studies, with higher signal to noise, are necessary for further progress.

\section{SUMMARY}

Based on observations of $\mathrm{CO}$ emission, we have detected $9.6 \pm 2.9 \times 10^{7} \mathscr{M}_{\odot}$ of counter-rotating molecular gas in the SB0 galaxy NGC 4546. The CO emission is strongest on the west side of the galaxy, contrary to eastern peak of the $\mathrm{H} \mathrm{I}$ emission. The ratio of molecular to atomic gas is less than normally seen in S0 galaxies, and seems to be a function of position in the galaxy. Both the atomic and molecular components are rotating opposite to the stars, and therefore are unlikely to be primordial. We suggest that it is likely that the gas was accreted when NGC 4546 absorbed a neighboring 
dwarf galaxy. The ratio of far-infrared luminosity to molecular mass, frequently used as a crude indicator of the "efficiency" of massive star formation, is similar to that in many quiescent spirals, indicating that whatever is happening now in NGC 4546 is not giving rise to a burst of star formation activity, and suggesting that the current situation in the galaxy is relatively stable.
L.J.S. acknowledges the support of the Max-PlanckGesellschaft through an MPG fellowship, and partial support of the North Atlantic Treaty Organization through Grant No. 910965. G.G. acknowledges partial support through a grant from the Ministry of University and Scientific Research under the program Astrofisica e Fisica Cosmica.

\section{REFERENCES}

Bertola, F., \& Bettoni, D. 1988, ApJ, 329, 102

Bertola, F., Bettoni, D., Buson, L., \& Zeilinger, W. W., 1990, in Dynamics \& Interactions of Galaxies, edited by R. Wielen (Springer, Berlin), p. 249

Bertola, F., Buson, L., \& Zeilinger, W. W. 1992, ApJ, 401, L79

Bettoni, D. 1984, The Messenger, 37, 17

Bettoni, D., Galletta, G., \& Osterloo, T. 1991, MNRAS, 248, 544

Braine, J., Combes, F., Casoli, F., Dupraz, C., Géring, M., Klein, U., Wielebinski, R., \& Brouillet, N. 1993, A\&AS, 97, 887

Braun, R., Walterbros, R. A. M., Kennicutt, Jr., R. C. 1992, Nature, 360, 442

Caldwell, N., Kirshner, R. P., \& Richstone, D. 1986 ApJ, 305, 136

Combes, F., Braine, J., Casoli, F., Gerin, M., \& van Driel, W. 1992, A\&A, 259, L65

Dettmar, R.-J., Jullien-Dettmar, M., \& Barteldrees, A., 1990, in The interstellar medium in external galaxies: Summary of contributed papers, edited by D. J. Hollenbach and H. A. Thronson (NASA, CP-3084), p. 246 Galletta, G. 1987, ApJ, 318, 531

Knapp, G. R., Guthathakurta, P., Kim, D. W., \& Jura, M. 1989, ApJS, 70, 329
Richter, O.-G., \& Huchtmeier, W. K. 1987, A\&AS, 68, 427

Rix, H-.W., \& Katz, N. 1991, in Warped Disks and Inclined Rings Around Galaxies, edited by S. Casertano, P. D. Sackett, and F. H. Briggs (Cambridge University Press, Cambridge), p. 112

Rubin, V. C., 1988, in Large Scale Motions in the Universe: A Vatican Study Week, edited by V. C. Rubin and C. V. Cohen (Princeton University Press, Princeton), p. 541

Sage, L. J. 1993, A\&A, 272, 173

Sage, L. J., Galletta, G. 1993, ApJ, 419, 544

Sage, L. J., \& Isbell, D. W. 1991, A\&A, 247, 320

Sage, L. J., Salzer, J. J., Loose, H.-H., \& Henkel, C. 1992, A\&A, 265, 19

Sage, L. J., Shore, S. N., \& Solomon, P. M. 1990, ApJ, 351, 422

Sage, L. J., \& Wrobel, J. M. 1989, ApJ, 344, 204

Schweizer, F., van Gorkom, J. H., \& Seitzer, P. 1989, ApJ, 338, 770

Solomon, P. M., Rivolo, A. R., Barrett, J. W., \& Yahil, A. 1987, ApJ, 319, 730

Solomon, P. M., \& Sage, L. J. 1988, ApJ, 334, 613

van Driel, W., \& Buta, R. 1993, PASJ, 45, L47 